\title{
Agents and medicines that reverse age related pseudocapillarization of liver sinusoidal endothelial cells in mice
}

\section{Nicholas J Hunt ${ }^{1,2}$, Glen Lockwood ${ }^{1,2}$, Hong Mao ${ }^{2,3}$, Alessandra Warren ${ }^{1}$, Peter Mccourt ${ }^{2,3}$, David Le Couteur $^{1,2}$, Victoria Cogger ${ }^{1,2}$}

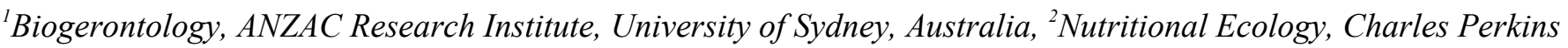
Centre, University of Sydney, Australia, ${ }^{3}$ Medical Biology, University of Tromso, Norway

Introduction: Fenestrations are pores within the liver sinusoidal endothelial cells (LSECs) that facilitate the transfer of substrates such as insulin and lipoproteins between blood and hepatocytes. With old age there is a loss of fenestrations referred to as pseudocapillarization which leads to hepatic insulin resistance and dyslipidemia. We are developing nanomedicines that target the LSEC in order to reverse age-related loss of fenestrations.

Methods: We investigated the effects of various agents on fenestrations in old compared to young C57/B16 mice. Isolated mice LSECs were incubated for 30 mins with Cytochalasin D (actin disruptor), 7-ketocholesterol (lipid raft disruptor), sildenafil, amlodipine and simvastatin (NO pathway promotors), 2,5-Dimethoxy-4-iodoamphetamine (DOI, serotonergic agent), bosentan (endothelin receptor inhibitor), TNF-related apoptosis-inducing ligand (TRAIL) and nicotinamide mononucleotide (NMN, NAD+ promotor). Cells were fixed and prepared for visualization under scanning electron microscopy (SEM) and direct stochastic optical reconstruction microscopy (dSTORM). SEM was used to examine fenestration porosity, diameter and frequency; dSTORM to examine actin and NO synthase densitometry.

Results: Old LSECs (18-24 mths, $\mathrm{n}=3$ ) had reduced fenestrations compared with young LSECs (4-6 mths, $\mathrm{n}=3$ ). In both young and old LSECs, porosity, diameter and frequency were increased with NMN $(\mathrm{P}<0.05)$; porosity and frequency only were increased by sildenafil, amlodipine, TRAIL, cytochalasin $\mathrm{D}$ and 7-ketocholesterol $(\mathrm{P}<0.05)$. In old mice only, bosentan and DOI increased fenestration porosity and frequency $(\mathrm{P}<0.05)$. Modification of the actin cytoskeleton was observed in all fenestration promoting agents with NO synthase promoted in sildenafil, amlodipine and TRAIL treatments $(\mathrm{P}<0.05)$.

Conclusion: We have shown that several agents and medicines targeting the NO pathway, actin and lipid raft disruption promote refenestration of aged mice LSECs to similar expression and patterns to young mice. Regulation of fenestrations may be performed by NO-dependent and independent pathways. Many of these agents demonstrate potential therapeutic use to treat age related pseudocapillarization in order to improve age-related insulin resistance and dyslipidemia. 